\title{
Octopus - Application test of a simple and effective tool for polishing slurry monitoring
}

\author{
Christian $\operatorname{Vogt}^{1 *}$, Oliver Faehnle, and Patrick Kochs ${ }^{2}$ and Rolf Rascher $^{1}$ \\ ${ }^{1}$ EUniversity of Applied Sciences, 94469, Deggendorf, Germany \\ ${ }^{2}$ EFisba AG, 9016 ST. Gallen, Switzerland
}

\begin{abstract}
In the digital age it has become a fundamental issue to monitor and control process parameters continuously and automatically. The monitoring of critical process parameters enables better quality at lower production costs and higher yields. In order to investigate the interactions between production quality and polishing parameters, we have developed a solution for automatic and reliable measurement of critical parameters. With this paper we first document the resolution and accuracy of the system compared to manual measuring methods currently in use.
\end{abstract}

The monitoring of critical process parameters enables better quality at lower production costs and higher yields. In polishing processes, the quality of the polishing agent is crucial. To ensure stable production processes, it is only logical to monitor the most important parameters:

$\mathrm{pH}$ value, density and temperature.

In many production lines, measurements are currently still carried out iteratively and manually, partly because automatic density measurement is particularly problematic and prone to errors when data is transferred manually.

In order to investigate the interactions between production quality and parameters, we have developed a solution for automatic and reliable measurements of the three parameters mentioned above as a first step.

The presented method works in situ and in process. The specially developed sensor for determining the density of the polishing agent provides a resolution of $0.001 \mathrm{~g} / \mathrm{ccm}$ with a maximum deviation of $4 \%$.

The system can be used for continuous monitoring of production processes. Furthermore, it is suitable for the documentation of experiments and the identification of correlations.

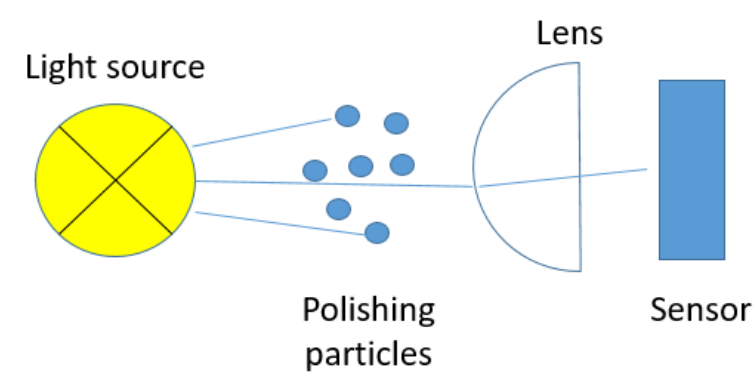

Fig. 1. Operating principle density sensor

The sensor for monitoring density measures the turbidity of the polishing agent and relies on a correlation of density. It is based on an intensity measurement of infrared radiation, whereby the degree of shading of the emitted beam by the polishing agent is measured and then the density is deduced (Fig. 1).

The system is kept very simple, extremely robust and yet has a high resolution. It can be used for several months without further calibration.

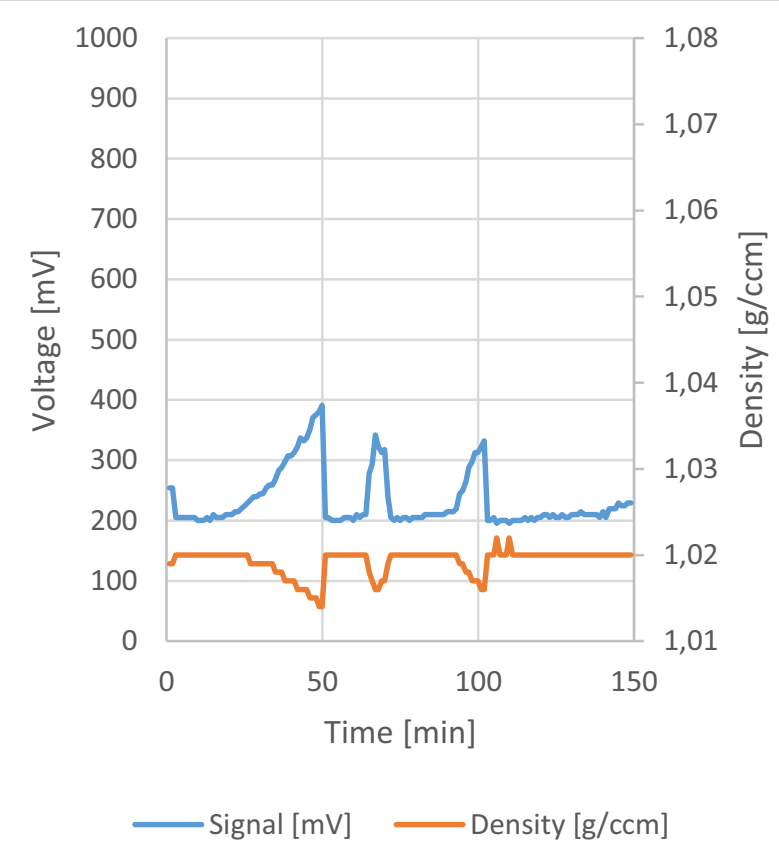

Fig. 2. Sample measurement with signal shape and density derived from it

\footnotetext{
*Corresponding author: $\underline{\text { Christian.vogt } @ \text { th-deg.de }}$
} 


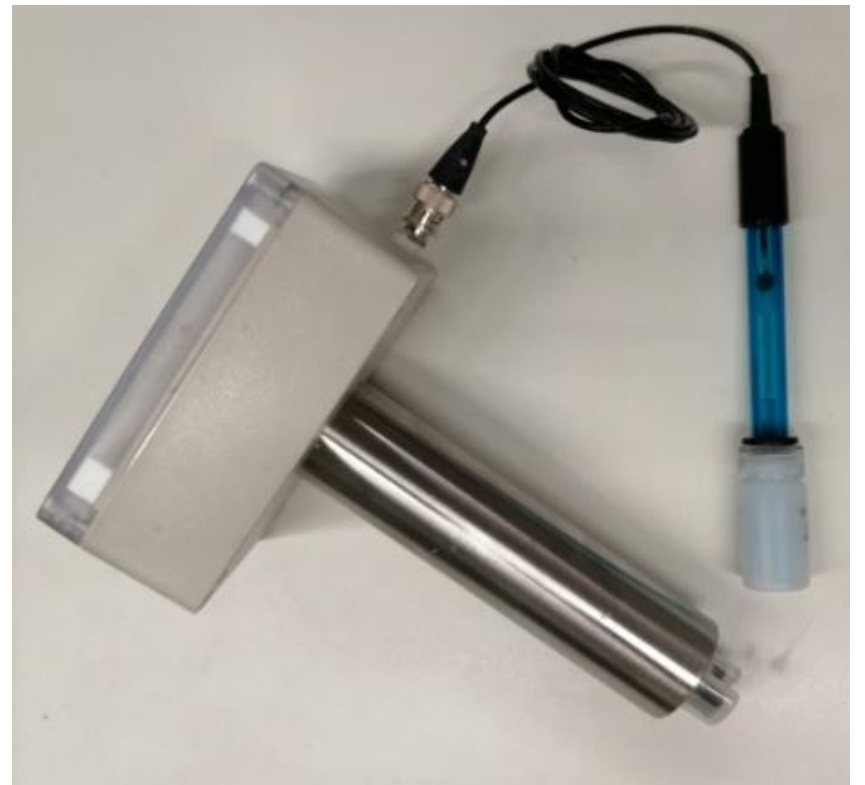

Fig. 3. Prototype with measurement technology for production monitoring, $\mathrm{pH}$-probe 